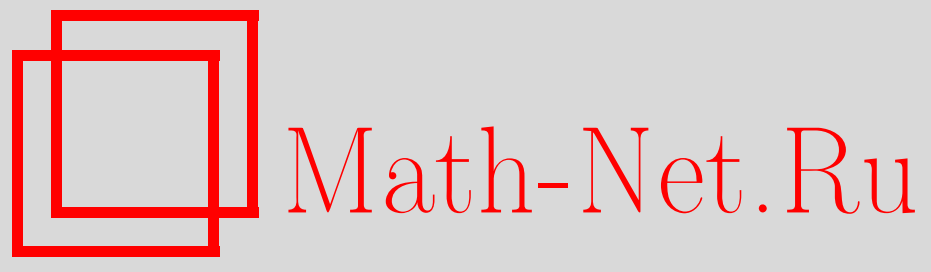

В. Л. Верещагин, Интегрируемая краевая задача для цепочки Вольтерра на полуоси, Матем. заметки, 2006, том 80, выпуск 5, 696-700

DOI: https://doi.org/10.4213/mzm3078

Использование Общероссийского математического портала Math-Net.Ru подразумевает, что вы прочитали и согласны с пользовательским соглашением http://www . mathnet.ru/rus/agreement

Параметры загрузки:

IP: 52.23 .180 .231

26 апреля 2023 г., 08:28:22

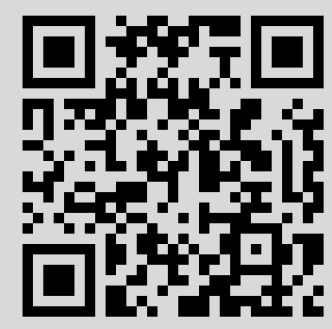




\section{ИНТЕГРИРУЕМАЯ КРАЕВАЯ ЗАДАЧА \\ ДЛЯ ЦЕПОЧКИ ВОЛЬТЕРРА НА ПОЛУОСИ}

\section{В. Л. Верещагин}

Исследованы квазипериодические (конечнозонные) решения цепочки Вольтерра, удовлетворяющие интегрируемому граничному условию на полуоси. Из множества общих конечнозонных решений выделены те, что отвечают краевой задаче, причем определяющее их условие имеет вид системы алгебраических уравнений.

Библиография: 7 названий.

1. Введение. Аналитическое изучение краевых задач для интегрируемых нелинейных уравнений в частных производных традиционно привлекает к себе внимание исследователей. Обычно подобные задачи рассматриваются либо в классе быстроубывающих при $|x| \rightarrow \infty$ функций, либо периодических по $x$. Из числа последних следует упомянуть конечнозонные решения краевых задач для нелинейного уравнения Шрёдингера [1], уравнений синус-Гордон [2] и Кортевега-де Фриза [3]. Данная работа преследует цель расширить круг изученных в этом аспекте систем и предъявить ограничения, выделяющие из общих конечнозонных решений цепочки Вольтерра те, которые удовлетворяют определенному граничному условию в точке $n=0$. Понятие интегрируемого краевого условия связано с ограничениями на решение в определенных точках, наложение которых не препятствует сохранению основных атрибутов интегрируемости (подробнее см., например, в [4]). Такие граничные условия уже получены для большей части наиболее важных интегрируемых систем; случай интегрируемых цепочек описан в работе [5]. Поэтому отыскание решений подобных краевых задач среди множества известных физически интересных классов представляет собой актуальную задачу. Данная статья предлагает решение указанной проблемы на примере цепочки Вольтерра.

2. Конечнозонные решения цепочки Вольтерра. Цепочка Вольтерра

$$
\dot{c}_{n}=c_{n}\left(c_{n+1}-c_{n-1}\right)
$$

где $c_{n}=c_{n}(t), n \in \mathbb{Z}, t \in \mathbb{R}$, является интегрируемой дифференциально-разностной системой, чьи конечнозонные решения впервые были описаны в работе [6]. Кратко укажем основные атрибуты метода. Обозначим $u_{n}=c_{2 n}, v_{n}=c_{2 n+1}$. Тогда

Работа выполнена при поддержке Российского фонда фундаментальных исследований, грант № 04-01-00190.

(C) В. Л. ВЕРЕщАГин, 2006 
система (1) примет вид

$$
\begin{aligned}
& \dot{u}_{n}=u_{n}\left(v_{n}-v_{n-1}\right), \\
& \dot{v}_{n}=v_{n}\left(u_{n+1}-u_{n}\right),
\end{aligned}
$$

эквивалентный так называемому представлению "нулевой кривизны"

$$
\dot{U}_{n}+U_{n} V_{n}-V_{n+1} U_{n}=0
$$

где

$$
U_{n}=\left(\begin{array}{cc}
\lambda-v_{n} & \sqrt{\lambda} u_{n} \\
-\sqrt{\lambda} & -u_{n}
\end{array}\right), \quad V_{n}^{(0)}=\left(\begin{array}{cc}
u_{n} & \sqrt{\lambda} u_{n} \\
-\sqrt{\lambda} & -\lambda+v_{n-1}
\end{array}\right),
$$

$\lambda$ - спектральный параметр. Решение соответствующих линейных уравнений (пара двухточечных функций Бейкера-Ахиезера)

$$
\Psi_{n}(t, \lambda)=\left(\psi_{n}(t, \lambda), \varphi_{n}(t, \lambda)\right)^{T}
$$

имеет вид

$$
\begin{aligned}
& \psi_{n}(t, \lambda)=\alpha_{n}^{1} \exp \left[n \int_{\lambda_{0}}^{\lambda} \Omega_{0}+t \int_{\lambda_{0}}^{\lambda} \Omega_{1}\right] \frac{\theta\left(\tau+A(\lambda)+D_{1}\right)}{\theta\left(A(\lambda)+D_{1}\right)}, \\
& \varphi_{n}(t, \lambda)=\alpha_{n}^{2} \exp \left[\left(n+\frac{1}{2}\right) \int_{\lambda_{0}}^{\lambda} \Omega_{0}+t \int_{\lambda_{0}}^{\lambda} \Omega_{1}\right] \frac{\theta\left(\tau+\frac{1}{2} U+A(\lambda)+D_{2}\right)}{\theta\left(A(\lambda)+D_{2}\right)},
\end{aligned}
$$

где $\tau=n U+t V, B$ - матрица периодов римановой поверхности $\Gamma$ рода $g:$

$$
w^{2}=R_{2 g+2}(\lambda)=\prod_{j=1}^{2 g+2}\left(\lambda-\lambda_{j}\right)
$$

векторы $U, V-b$-периоды нормированных дифференциалов второго рода $\Omega_{0}, \Omega_{1}$ соответственно:

$$
\Omega_{0}=-\frac{\lambda^{g} d \lambda}{\sqrt{R_{2 g+2}(\lambda)}}+\sum_{j=1}^{g} d_{j}^{0} \omega_{j}, \quad \Omega_{1}=\frac{-\lambda^{g+1}+\frac{1}{2} \Lambda \lambda^{g}}{\sqrt{R_{2 g+2}(\lambda)}} d \lambda+\sum_{j=1}^{g} d_{j}^{1} \omega_{j},
$$

$d_{j}^{0,1}-$ константы, $\omega_{j}$ - нормированные голоморфные дифференциалы на римановой поверхности Г. Полином

$$
R_{2 g+2}(\lambda)=\operatorname{det} V_{n}^{(g)}-\frac{1}{4}\left(\operatorname{Sp} V_{n}^{(g)}\right)^{2},
$$

$g$ - номер оператора $V_{n}$ в интегрируемой иерархии, $\Lambda=\sum_{j=1}^{2 g+2} \lambda_{j}$;

$$
A(\lambda)=\left(\int_{\lambda_{0}}^{\lambda} \omega_{1}, \ldots, \int_{\lambda_{0}}^{\lambda} \omega_{g}\right)^{T}
$$

- отображение Абеля, $\theta$ - тета-функция римановой поверхности $\Gamma$ рода $g$ :

$$
\theta(Z \mid B)=\sum_{N \in \mathbb{Z}^{g}} \exp \left(\frac{1}{2}\langle N, B\rangle+\langle Z, N\rangle\right)
$$


$D_{1} \neq D_{2} \in \mathbb{C}^{g}$ - постоянные векторы,

$$
\left(\alpha_{n}^{1}\right)^{2}=\frac{\theta\left(\tau+D_{1}-U\right) \theta\left(D_{1}\right)}{\theta\left(\tau+D_{1}\right) \theta\left(D_{1}-U\right)}, \quad\left(\alpha_{n}^{2}\right)^{2}=\frac{\theta\left(\tau-U+D_{2}\right) \theta\left(D_{2}\right)}{\theta\left(\tau+D_{2}\right) \theta\left(D_{2}-U\right)} ;
$$

сами же решения системы (2) выводятся из условий разложения в окрестности одной из существенных особых точек:

$$
\begin{gathered}
v_{n}^{1 / 2} \frac{\varphi_{n}}{\psi_{n}}=\sqrt{\lambda}\left(1+O\left(\lambda^{-1}\right)\right), \quad u_{n}^{1 / 2} \frac{\psi_{n}}{\varphi_{n-1}}=\sqrt{\lambda}\left(1+O\left(\lambda^{-1}\right)\right), \quad \lambda \rightarrow \infty_{+}, \\
u_{n}=\frac{\theta\left(D_{1}\right) \theta\left(D_{1}-U\right)}{\theta\left(D_{2}-U\right) \theta\left(D_{2}\right)} \frac{\theta\left(\tau+D_{2}-2 U\right) \theta\left(\tau+D_{1}\right)}{\theta\left(\tau+D_{2}-U\right) \theta\left(\tau+D_{1}-U\right)}, \\
v_{n}=\frac{\theta\left(D_{2}\right) \theta\left(D_{2}-U\right)}{\theta\left(D_{1}-U\right) \theta\left(D_{1}\right)} \frac{\theta\left(\tau+D_{1}-U\right) \theta\left(\tau+D_{2}\right)}{\theta\left(\tau+D_{2}-U\right) \theta\left(\tau+D_{1}\right)} .
\end{gathered}
$$

Отметим, что вся описанная схема редуцируется к указанной в работе [6] схеме системы при условии $D_{2}-D_{1}=\frac{1}{2} U$ и соответственно равенства одной из точек ветвления римановой поверхности Г нулю.

3. Краевая задача и конечнозонные решения. Авторы статьи [5] привели список интегрируемых краевых условий для цепочки Вольтерра, из которых

$$
u_{0}+v_{0}+\mu=0
$$

где $\mu$ - некоторая константа, следует считать наиболее простым из нетривиальных. Поставим задачу об отыскании конечнозонных решений цепочки Вольтерра, удовлетворяющих условию (9). Используемый далее метод базируется на известном наблюдении А.И. Бобенко, заключающемся в том, что интегрируемые граничные условия можно записать как условия треугольности оператора $V_{n}$. Это обстоятельство позволяет использовать известный вид решений (5) линейной системы и довольно просто выделить решения краевой задачи.

Первым оператором $V_{n}^{j}$ из иерархии цепочки Вольтерра, ведущим к нетривиальным решениям, является следующий:

$$
V_{n}^{(1)}=\left(\begin{array}{cc}
-\frac{1}{2} \lambda^{2}-\lambda\left(u_{n}+\frac{a}{2}\right)-u_{n}\left(v_{n-1}+u_{n}+v_{n}+a\right) & -\sqrt{\lambda} u_{n}\left(\lambda+u_{n}+v_{n}+a\right) \\
\lambda^{3 / 2}+\sqrt{\lambda}\left(v_{n-1}+u_{n}+a\right) & \frac{1}{2} \lambda^{2}+\lambda\left(u_{n}+\frac{a}{2}\right)-v_{n-1}\left(u_{n-1}+v_{n-1}+u_{n}+a\right)
\end{array}\right),
$$

где $a$ - произвольный постоянный параметр. Очевидно, что выполнение условия (9) дает равенство

$$
\left.\left(V_{n}^{(1)}\right)_{12}(n, t, \lambda)\right|_{n=0, \lambda=\mu-a}=0 \quad \text { для всех } t ;
$$

следовательно, среди конечнозонных функций Бейкера-Ахиезера (5) найдутся отвечающие тривиальному решению линейного уравнения и

$$
\psi_{0}(t, \mu-a)=0 \quad \text { для всех } t .
$$

Таким образом, задача сводится к описанию параметров римановой поверхности Г и функции Бейкера-Ахиезера, отвечающих условию на нули тета-функции:

$$
\left.\theta\left(\tau+A\left(P_{0}\right)+D_{1}\right)\right|_{n=0}=0 \quad \text { для всех } t, \quad P_{0}=\pi^{-1}(\mu-a),
$$


$\pi$ - оператор проекции римановой поверхности на комплексную плоскость. Потребуем от $\Gamma(w, \lambda)$ выполнения инволюции $\nu:(\omega, \lambda) \rightarrow(\omega,-\lambda)$, род ее $g$ будем считать четным: $g=2 k, k=1,2, \ldots$. Для $k=1$ имеем картину разрезов на листах $\Gamma$, указанную на рис. 1.

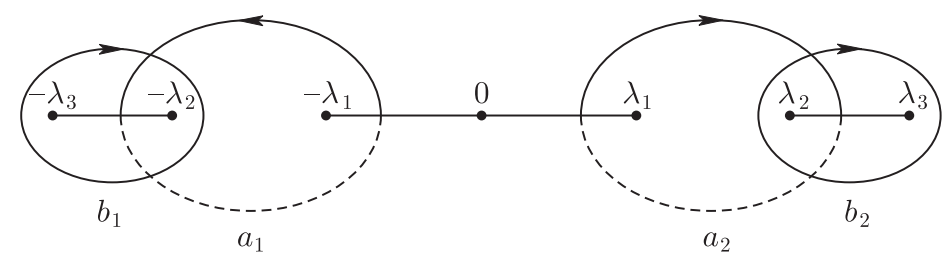

Рис. 1. Базис циклов на римановой поверхности

Выбрав канонический базис циклов, в силу симметрии Г и условий четности дифференциалов $\Omega_{0,1}$ получаем соотношения на их $b$-периоды:

$$
U_{j}=U_{j+k}, \quad V_{j}=-V_{j+k}, \quad j=1, \ldots, k .
$$

Известно [7], что из-за симметрии римановой поверхности соответствующая тетафункция $\theta(\lambda \mid B)$ редуцируется к тета-функциям меньшей размерности с характеристиками

$$
\theta\left(\begin{array}{c}
z_{1} \\
z_{2}
\end{array} \mid B\right)=\sum_{\delta \in \frac{1}{2} \mathbb{Z}_{2}^{k}} \theta[\delta, 0]\left(z_{1}+z_{2} \mid 2 \Pi\right) \theta[\delta, 0]\left(z_{1}-z_{2} \mid 2 T\right),
$$

где

$$
\begin{aligned}
\theta[\alpha, \beta](z) & =\exp \left(\frac{1}{2}\langle B \alpha, \alpha\rangle+\langle z+2 \pi i \beta, \alpha\rangle\right) \theta(z+2 \pi i \beta+B \alpha) \\
& =\sum_{N \in \mathbb{Z}^{g}} \exp \left(\frac{1}{2}\langle B(N+\alpha), N+\alpha\rangle+\langle z+2 \pi i \beta, N+\alpha\rangle\right)
\end{aligned}
$$

- функция с характеристиками $\alpha, \beta$, матрица $T$ определена в [7],

$$
\Pi_{i j}=\oint_{b_{i}} \omega_{i}+\omega_{j+k}, \quad i, j=1, \ldots, k .
$$

Разложив таким образом тета-функцию (13) в сумму (15), получим, что в каждом слагаемом один из сомножителей в силу (14) не зависит от $t$ (это так называемые тета-константы). Таким образом, используя симметрию, ищем нули тета-констант:

$$
\theta\left(\tilde{A}\left(P_{0}\right)+\tilde{D}+4 \Pi \delta \mid 2 \Pi\right)=0, \quad \delta \in \frac{1}{2} \mathbb{Z}_{2}^{k},
$$

где $\tilde{A}^{j}=A^{j}+A^{j+k}, \tilde{D}^{j}=D_{1}^{j}+D_{1}^{j+k}, \quad j=1,2, \ldots k, \quad \theta$ - тета-функция рода $k . \mathrm{B}$ соответствии с известной теоремой Римана нули тета-функции допускают параметрическое представление в виде

$$
e=\tilde{A}\left(P_{1}\right)+\cdots+\tilde{A}\left(P_{k-1}\right)+K,
$$


где $P_{1}, \ldots, P_{k-1}-$ произвольные точки римановой поверхности $\Gamma$,

$$
K_{j}=\pi_{i}+\Pi_{j j}-\frac{1}{2 \pi i} \sum_{l \neq j}\left(\oint_{a_{l}}\left(\omega_{l}+\omega_{l+k}\right)(P) \int_{P_{0}}^{P} \omega_{j}\right), \quad j=1, \ldots, k,
$$

- вектор римановых констант. Следовательно, получаем следующее утверждение.

Теорема. Решение цепочки Волътерра удовлетворяет краевому условию (9), если величины D удовлетворяют равенству

$$
\tilde{D} \equiv \sum_{j=1}^{k-1} \tilde{A}\left(P_{j}\right)-\tilde{A}\left(P_{0}\right)+K
$$

$P_{0}=\pi^{-1}(\mu-a)$. Знак '三' означает равенство по модулю решетки периодов тетафункции, $P_{1}, \ldots, P_{k-1}$ - произвольные точки римановой поверхности Г.

Автор выражает признательность И. Т. Хабибуллину за ценные консультации.

\section{СПИСОК ЦИТИРОВАННОЙ ЛИТЕРАТУРЫ}

[1] Р. Ф. Бикбаев, А. Р. Итс, “Алгеброгеометрические решения краевой задачи для нелинейного уравнения Шрёдингера”, Матем. заметки, 45:5 (1989), 3-9.

[2] Р. Ф. Бикбаев, В. О. Тарасов, "Неоднородная краевая задача на полуоси и на отрезке для уравнения Sine-Gordon", Алгебра и анализ, 3:4 (1991), 78-92.

[3] В.Э. Адлер, И. Т. Хабибуллин, А. Б. Шабат, "Краевая задача для уравнения КдФ на полуоси", ТМФ, 110:1 (1997), 98-113.

[4] Е.К. Склянин, "Граничные условия для интегрируемых уравнений", Функцион. анализ и его прилож., 21:2 (1987), 86-87.

[5] В.Э. Адлер, И. Т. Хабибуллин, "Граничные условия для интегрируемых цепочек", Функиион. анализ и его прилож., 31:2 (1997), 1-14.

[6] А. П. Веселов, "Интегрирование стационарной задачи для классической спиновой цепочки", ТМФ, 71:1 (1987), 154-159.

[7] J. Fay, Theta-Functions on Riemann Surface, Lecture Notes in Math., 352, SpringerVerlag, New York, 1973. 